\title{
Hollow nanocylinder: Multisubband superconductivity induced by quantum confinement
}

\author{
Yajiang Chen, A. A. Shanenko, ${ }^{*}$ and F. M. Peeters \\ Departement Fysica, Universiteit Antwerpen, Groenenborgerlaan 171, B-2020 Antwerpen, Belgium \\ (Received 26 November 2009; revised manuscript received 11 March 2010; published 26 April 2010)
}

\begin{abstract}
Quantization of the transverse electron motion in high-quality superconducting metallic nanowires and nanofilms results in the formation of well-distinguished single-electron subbands. They shift in energy with changing thickness, which is known to cause quantum-size superconducting oscillations. The formation of multiple subbands results in a multigap structure induced by the interplay between quantum confinement and Andreev mechanism. We investigate multisubband superconductivity in a hollow nanocylinder by numerically solving the Bogoliubov-de Gennes equations. When changing the inner radius and thickness of the hollow nanocylinder, we find a crossover from an irregular pattern of quantum-size superconducting oscillations, typical of nanowires, to an almost regular regime, specific for superconducting nanofilms. At this crossover the multigap structure becomes degenerate. The ratio of the critical temperature to the energy gap increases and approaches its bulk value while being reduced by $20-30 \%$ due to Andreev-type states driven by quantum confinement in the irregular regime.
\end{abstract}

DOI: 10.1103/PhysRevB.81.134523

PACS number(s): 74.45.+c, 74.62.- c, 74.78.-w

\section{INTRODUCTION}

High-quality metallic superconducting nanofilms and nanowires are now available due to recent advances in nanofabrication technologies. In particular, ultrathin singlecrystalline flat islands of $\mathrm{Pb}$ can now be routinely fabricated with atomically uniform thickness down to few monolayers and lateral dimensions from $100-200 \mathrm{~nm}$ to $1-2 \mu \mathrm{m} .{ }^{1-9}$ Such nanofilms are nearly impurity free (see, e.g., Ref. 7) but grown on a disordered wetting layer (about a few monolayers of $\mathrm{Pb}$ ) on silicon substrate). This disordered interface controls the electron mean-free path that is approximately twice the nanofilm thickness (see, e.g., Ref. 3). As a result, clear signatures of the quantized transverse electron spectrum (quantum-well states) are observed in tunneling data $^{1,3,6,9}$ and, so, the conduction band splits up into a series of subbands. The single-crystalline nanofilms are low resistive and show no significant suppression of superconductivity due to fluctuations or disorder (see discussion in Ref. 3). It was recently found ${ }^{9}$ that superconductivity survives even in $\mathrm{Pb}$ nanofilms with an extremely small thickness of 2 monolayers.

Similar steadiness of superconductivity is observed in high-quality nanowires, both single crystalline (Sn, see Refs. 10-12) and made of strongly coupled grains (Al, Refs. 13-15). Here signatures of the superconducting state are found for diameters down to $5-10 \mathrm{~nm} \cdot{ }^{14,15}$ The electron mean-free path is estimated to be of about the nanowire thickness (see, e.g., Refs. 15 and 16). Thus, the formation of distinguished single-electron subbands can be expected due to the transverse quantization.

Superconductivity in the presence of multiple bands can in general lead to a wealth of unusual superconducting phenomena, such as, e.g., Leggett's collective mode, ${ }^{17}$ Tanaka's soliton, ${ }^{18-20}$ and fractional flux. ${ }^{21,22}$ The reason is that the pair condensate can have different phases in different bands, which brings new degrees of freedom. The multisubband structure induced by quantum confinement results in additional important possibilities. Formed due to the transverse quantization, subbands move in energy when changing the nanowire/nanofilm thickness. In particular, this leads to quantum-size oscillations accompanied by significant enhancements each time when the bottom of a new singleelectron subband passes through the Fermi surface, i.e., the quantum-size superconducting resonance. ${ }^{23}$ Recently, quantum-size oscillations of the critical superconducting temperature $T_{c}$ and critical magnetic field $H_{c 2}$ have been observed in $\mathrm{Pb}$ nanofilms. ${ }^{1,4,9}$ Furthermore, the results of a numerical self-consistent investigation of the Bogoliubov-de Gennes (BdG) equations in Ref. 24 shows that the superconducting resonances are responsible for a systematic shift-up of $T_{c}$ observed in aluminum and tin nanowires with a decrease in the cross section. ${ }^{10,11,13-15}$ Other interesting effects can be expected in high-quality nanofilms and nanowires due to the formation of the multisubband structure, e.g., Andreev-type states induced by quantum confinement ${ }^{25}$ and a cascade character of the superconductor-to-normal transition driven by a magnetic field/supercurrent. ${ }^{26,27}$ All this makes it possible to expect that the multisubband structure realized due to quantum confinement can open new prospects of tailoring superconducting properties by changing the main geometrical parameters of nanoscale superconductors.

Despite recent progress, many details of the superconducting state in the presence of the restricted dimensionality and multiple subbands remain unexplored. In particular, this concerns superconducting systems with an annular confining geometry, e.g., a hollow nanocylinder or nanoring, representing a promising choice for future superconducting nanodevices (see, e.g., recent papers on nanorings ${ }^{28-31}$ ). In the present paper we show that the superconducting condensate, energy gap, and critical temperature of a hollow metallic nanocylinder (with inner radius $R$ and thickness $d$ ) can be strongly modified by changing $R$ and $d$. The formation of multiple subbands results in quantum-size superconducting oscillations and in a multigap structure driven by the interplay of quantum confinement with Andreev mechanism. Quantum-size oscillations of the basic superconducting quantities as function of $d$ exhibit a qualitative change from an irregular regime at small inner radii to almost regular 
oscillations for large $R$. At this crossover the multigap structure becomes degenerate. The ratio of the critical temperature to the energy gap increases and approaches its bulk value while being reduced by $20-30 \%$ due to Andreev-type states in the irregular regime. Our investigation is based on a numerical self-consistent solution of the $\mathrm{BdG}$ equations in the clean limit.

The paper is organized as follows. In Sec. II, we outline the formalism of the BdG equations and discuss how to construct Anderson's approximate solution to the BdG equations for a hollow metallic nanocylinder. In Sec. III we demonstrate that results of a numerical solution of the BdG equations are in very good agreement with those of Anderson's approximation. This allows us to abandon time-consuming numerical study of the BdG equations in favor of solving the BCS-type equation. Then, the basic superconducting properties, i.e., the order parameter, energy gap, and critical temperature, are investigated as function of the main geometrical parameters $R$ and $d$.

\section{FORMALISM}

\section{A. Bogoliubov-de Gennes equations}

In the absence of a magnetic field the $\mathrm{BdG}$ equations can be written in the form ${ }^{32}$

$$
\begin{aligned}
& E_{n} u_{n}(\mathbf{r})=\hat{H}_{e} u_{n}(\mathbf{r})+\Delta(\mathbf{r}) v_{n}(\mathbf{r}), \\
& E_{n} v_{n}(\mathbf{r})=\Delta(\mathbf{r}) u_{n}(\mathbf{r})-\hat{H}_{e} v_{n}(\mathbf{r}),
\end{aligned}
$$

where $\Delta(\mathbf{r})$ is the superconducting order parameter (taken as real), $E_{n}$ is the quasiparticle spectrum with $n$ the set of the relevant quantum numbers, $u_{n}(\mathbf{r})$ and $v_{n}(\mathbf{r})$ are the particlelike and holelike wave functions, and $\hat{H}_{e}=-\frac{\hbar^{2}}{2 m_{e}} \nabla^{2}-E_{F}$, with $m_{e}$ the electron band mass and $E_{F}$ the Fermi energy in the normal state.

The BdG equations have to be solved in a self-consistent manner, together with the self-consistency relation given by

$$
\Delta(\mathbf{r})=g \sum_{n} u_{n}(\mathbf{r}) v_{n}^{*}(\mathbf{r})\left(1-2 f_{n}\right),
$$

where $f_{n}=f\left(E_{n}\right)$ is the Fermi function and $g>0$ is the coupling constant controlling the Cooper pairing. The particlelike and holelike amplitudes in Eq. (2) obey the normalization condition

$$
\int d^{3} r\left(\left|u_{n}(\mathbf{r})\right|^{2}+\left|v_{n}(\mathbf{r})\right|^{2}\right)=1 .
$$

The sum in Eq. (2) is over the electron states with positive quasiparticle energy. In addition, to remedy the ultraviolet divergence in the self-consistency relation, we need to invoke a cutoff resulting in taking only states with singleelectron energy $\xi_{n}$ within the Debye window around the Fermi level, i.e., $\left|\xi_{n}\right|<\hbar \omega_{D}$ with

$$
\xi_{n}=\int d^{3} r\left[u_{n}^{*}(\mathbf{r}) \hat{H}_{e} u_{n}(\mathbf{r})+v_{n}^{*}(\mathbf{r}) \hat{H}_{e} v_{n}(\mathbf{r})\right] .
$$

Due to the grand-canonical character of the BdG formalism, the chemical potential (equal to $E_{F}$ ) enters the basic equa- tions and, so, the single-electron energy in Eq. (4) is measured from the Fermi level. For a given mean electron density $n_{e}, E_{F}$ is determined from

$$
n_{e}=\frac{2}{V} \sum_{n} \int d^{3} r\left[\left|u_{n}(\mathbf{r})\right|^{2} f_{n}+\left|v_{n}(\mathbf{r})\right|^{2}\left(1-f_{n}\right)\right]
$$

with $V=\pi d(d+2 R) L$ the volume of a hollow nanocylinder with length $L$, where $L \rightarrow \infty$.

For the chosen geometry, the superconducting order parameter depends only on $\rho(\rho, \varphi, z$ are the cylindrical coordinates), i.e., $\Delta(\mathbf{r})=\Delta(\rho)$. It implies that we restrict ourselves to the case of zero net angular momentum of the condensate (no vortex) and zero net momentum along the $z$ axis (no longitudinal supercurrent). States with a finite net angular momentum have a much higher kinetic energy in the case of interest. The particlelike and holelike amplitudes can be represented as

$$
u_{n}(\mathbf{r})=u_{n}(\rho) \frac{e^{l m \varphi}}{\sqrt{2 \pi}} \frac{e^{i k z}}{\sqrt{L}}, \quad v_{n}(\mathbf{r})=v_{n}(\rho) \frac{e^{i m \varphi}}{\sqrt{2 \pi}} \frac{e^{i k z}}{\sqrt{L}},
$$

where $n=\{j, m, k\}, j$ is the radial quantum number controlling the number of zeros of $u_{n}(\rho)$ and $v_{n}(\rho)$ for $R<\rho<R$ $+d, m$ is the azimuthal quantum number, and $k$ is the wave vector of the quasifree electron motion parallel to the nanocylinder. Due to transverse quantum confinement

$$
\begin{aligned}
& \left.u_{n}(\mathbf{r})\right|_{\rho=R}=\left.u_{n}(\mathbf{r})\right|_{\rho=R+d}=0, \\
& \left.v_{n}(\mathbf{r})\right|_{\rho=R}=\left.v_{n}(\mathbf{r})\right|_{\rho=R+d}=0,
\end{aligned}
$$

while periodic boundary conditions are used in the longitudinal and azimuthal directions.

For a numerical solution of the BdG equations, we expand the particlelike and holelike radial wave functions $u_{j m k}(\rho)$ and $v_{j m k}(\rho)$ in terms of the single-electron radial wave functions $\psi_{j m}(\rho)$, i.e.,

$$
\begin{aligned}
& u_{j m k}(\rho)=\sum_{j^{\prime}} U_{j m k, j^{\prime}} \psi_{j^{\prime} m}(\rho), \\
& v_{j m k}(\rho)=\sum_{j^{\prime}} V_{j m k, j^{\prime}} \psi_{j^{\prime} m}(\rho) .
\end{aligned}
$$

We chose $u_{j m k}(\rho)$ and $v_{j m k}(\rho)$ real and, so, the expansion coefficients $U_{j m k, j^{\prime}}$ and $V_{j m k, j^{\prime}}$ are real, as well. Then, inserting Eq. (8) into Eq. (1), the expansion coefficients $U_{j m k, j^{\prime}}$ and $V_{j m k, j^{\prime}}$ can be calculated together with $E_{j m k}$ by means of diagonalizing the relevant matrix. Iterations are invoked to realize a self-consistent solution. The wave function $\psi_{j m}(\rho)$ is given by the equation

$$
\left[-\frac{1}{\rho} \frac{\partial}{\partial \rho} \rho \frac{\partial}{\partial \rho}+\frac{m^{2}}{\rho^{2}}\right] \psi_{j m}(\rho)=\chi_{j m}^{2} \psi_{j m}(\rho),
$$

where $\hbar^{2} \chi_{j m}^{2} /\left(2 m_{e}\right)$ stands for the contribution of the radial and azimuthal motion to the single-electron energy. Due to the quantum-confinement boundary conditions given by Eq. (7), one should set 


$$
\left.\psi_{j m}(\rho)\right|_{\rho=R}=\left.\psi_{j m}(\rho)\right|_{\rho=R+d}=0 .
$$

From Eqs. (9) and (10) it follows that $\psi_{j m}(\rho)$ can be written as

$$
\psi_{j m}(\rho)=\frac{1}{\sqrt{\mathcal{M}}}\left[Y_{m}\left(\chi_{j m} R\right) J_{m}\left(\chi_{j m} \rho\right)-J_{m}\left(\chi_{j m} R\right) Y_{m}\left(\chi_{j m} \rho\right)\right],
$$

where $J_{m}(x)$ and $Y_{m}(x)$ are the Bessel functions of the first and second kind of $m$ th order and the normalization constant $M$ is given by

$$
\mathcal{M}=\left.\frac{\rho}{2}\left[Y_{m}\left(\chi_{j m} R\right) J_{m+1}\left(\chi_{j m} \rho\right)-J_{m}\left(\chi_{j m} R\right) Y_{m+1}\left(\chi_{j m} \rho\right)\right]\right|_{\rho=R} ^{\rho=R+d} .
$$

The transverse single-electron energy $\chi_{j m}=\chi_{j m}(R, d)$ is a solution of

$$
\left|\begin{array}{cc}
J_{m}\left(\chi_{j m} R\right) & Y_{m}\left(\chi_{j m} R\right) \\
J_{m}\left[\chi_{j m}(R+d)\right] & Y_{m}\left[\chi_{j m}(R+d)\right]
\end{array}\right|=0,
$$

which follows from Eq. (10). Notice that generally, $\chi_{j m}$ has a complicated dependence on the geometrical parameters $R$ and $d$. However, in most cases $\chi_{j m}$ is controlled by $d$ : for $R \rightarrow 0$ we get $\chi_{j m} \propto 1 / d$, and a similar dependence is found for $R \rightarrow \infty$ or $d \rightarrow 0$. This can also be seen from the Heisenberg uncertainty principle because $\chi_{j m}$ can be interpreted as an average modulus of the transverse electron momentum.

Below we consider material parameters typical of aluminum: $\hbar \omega_{D}=32.31 \mathrm{meV} ; g N(0)=0.18$, with $N(0)$ the bulk density of states at the Fermi level. Notice that the BdG equations given by Eq. (1) are written in the parabolic band approximation. This implies working with an effective Fermi level (for more details, see Ref. 33). For aluminum $E_{F}$ $=0.9 \mathrm{eV}$, which was obtained through a good agreement with the experimental data in Ref. 24. With decreasing thickness, as results from Eq. (5), $E_{F}$ systematically shifts up from $0.9 \mathrm{eV}$ due to quantum-size effects. Such a shift can be appreciable (about 20\%) for $d \sim 1-2 \mathrm{~nm}\left(E_{F}\right.$ is almost insensitive to $R$ ). In addition, each time when a new singleelectron subband passes through the Fermi surface, $E_{F}$ exhibits a kink similar to the dependence of $E_{F}$ on the radius of a cylindrical nanowire. ${ }^{33}$ For the chosen parameters the bulk BCS coherence length and energy gap are $\xi_{0}$ $=1.5 \mu \mathrm{m} \gg R, d$ and $\Delta_{\text {bulk }}=0.25 \mathrm{meV}$, respectively. The particular choice of $\mathrm{Al}$ does not influence our conclusions. For instance, quantum-size oscillations in a Sn hollow nanocylinder will also be present but with an amplitude that is a factor of 2 smaller. In addition, by the same reason, we do not address any issue concerning a thickness-dependent change in the electron-phonon coupling. Its increase due to the surface softening of the phonon spectrum ${ }^{34}$ or a possible substrate-induced decrease ${ }^{1,8}$ can result in quantitative effects but do not alter the qualitative picture.

\section{B. Anderson's solution}

When the Cooper pairing between electrons from different single-electron subbands is insignificant we can invoke
Anderson's approximate solution to the BdG equations. ${ }^{35} \mathrm{~A}$ significant advantage of such an approximation is its semianalytical character, which makes it possible to reduce the $\mathrm{BdG}$ equations to a BCS-type self-consistent equation whose numerical solution is much less time consuming.

Anderson's solution means that $u_{j m k}(\rho)$ and $v_{j m k}(\rho)$ are taken to be proportional to $\psi_{j m}(\rho)$ given by Eq. (11), i.e., the coefficients of the expansion in Eq. (8) are assumed to be of the form ${ }^{26}$

$$
U_{j m k, j^{\prime}}=\mathcal{U}_{j m k} \delta_{j j^{\prime}}, \quad V_{j m k}=\mathcal{V}_{j m k} \delta_{j j^{\prime}}
$$

with $\delta_{j j^{\prime}}$ the discrete delta function. In this case the singleelectron energy reads

$$
\xi_{j m k}=\frac{\hbar^{2}}{2 m_{e}}\left(\chi_{j m}^{2}+k^{2}\right)-E_{F} .
$$

Inserting Eq. (14) into Eq. (1) and, then, multiplying the resulting expressions by $\psi_{j m}(\rho)$, one can make a straightforward integration, which yields

$$
E_{j m k}=\sqrt{\xi_{j m k}^{2}+\Delta_{j m}^{2}}
$$

and

$$
U_{j m k} V_{j m k}=\frac{\Delta_{j m}}{2 \sqrt{\xi_{j m k}^{2}+\Delta_{j m}^{2}}}
$$

with $\Delta_{j m}$ given by

$$
\Delta_{j m}=\int_{R}^{R+d} d \rho \rho \psi_{j m}(\rho) \Delta(\rho) \psi_{j m}(\rho) .
$$

Making use of Eq. (2) together with Eqs. (14) and (17), one obtains the following BCS-type self-consistent equation,

$$
\Delta_{j^{\prime} m^{\prime}}=-\sum_{j m k} J_{j^{\prime} m^{\prime}, j m} \frac{\Delta_{j m}}{2 E_{j m k}}\left[1-2 f\left(E_{j m k}\right)\right]
$$

with the interaction matrix of the form

$$
J_{j^{\prime} m^{\prime}, j m}=-\frac{g}{2 \pi L} \int_{R}^{R+d} d \rho \rho \psi_{j^{\prime} m^{\prime}}^{2}(\rho) \psi_{j m}^{2}(\rho) .
$$

As mentioned above, Eq. (14) is a good approximation when there is no significant pairing between electrons from different subbands (i.e., nondegenerating subbands with different $j$ and $|m|)$. This is correct when the intersubband energy spacing $\delta_{\text {sub }}$ is compatible with or larger than the Debye energy $\hbar \omega_{D}$ : in such a case we have a negligible probability to pick up two single-electron states in the Debye window, i.e., $\left|\xi_{j m k}\right|<\hbar \omega_{D}$, with different transverse energies and opposite longitudinal momenta. The above energy spacing can be taken as $\delta_{\text {sub }} \sim \frac{\hbar^{2}}{2 m_{e}}\left(\chi_{j+1, m}^{2}-\chi_{j m}^{2}\right)$. According to the remark about the dependence of $\chi_{j m}$ on $R$ and $d$ after Eq. (13), one can approximate $\delta_{\text {sub }}$ as $\delta_{\text {sub }} \sim \frac{\hbar^{2}}{2 m_{e}} \frac{\pi^{2}}{d^{2}}$, which is the intersubband energy spacing for nanofilms. Using such a simplification, one obtains that Anderson's approximation given by Eq. (14) is good enough when 

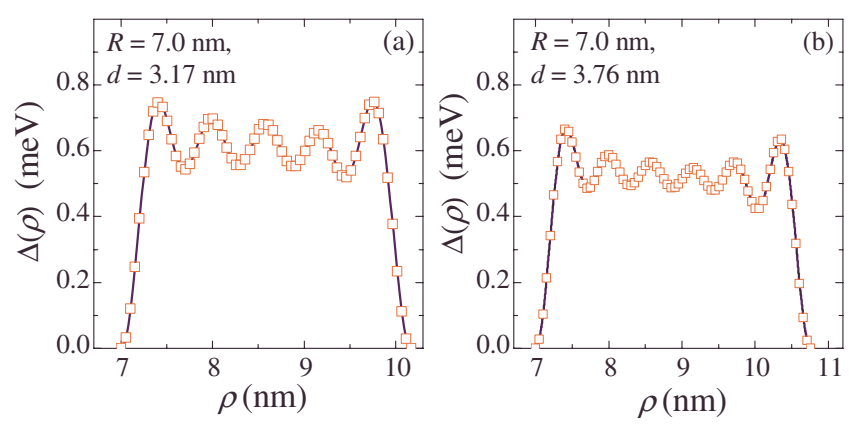

FIG. 1. (Color online) The order parameter $\Delta(\rho)$ (at $T=0$ ) as calculated from the full BdG equations (solid curve) and from Anderson's approximation (open squares): (a) $R=7 \mathrm{~nm}$ and $d$ $=3.17 \mathrm{~nm}$; and (b) $R=7 \mathrm{~nm}$ and $d=3.76 \mathrm{~nm}$.

$$
d \lesssim \pi \sqrt{\frac{\hbar}{2 m_{e} \omega_{D}}} .
$$

With typical metallic values $\hbar \omega_{D} \approx 10-30 \mathrm{meV}$, it follows from Eq. (21) that $d \$ 4-6 \mathrm{~nm}$. In Sec. III we check this expectation through a numerical analysis. Note that Eq. (21) is a necessary but not a sufficient condition for the accuracy of Anderson's approximation, Eq. (14). It can be poor when time-reversal symmetry is broken, e.g., in the presence of a magnetic field. In this case the orientation of a magnetic field is of importance. For instance, if it is parallel to the nanocylinder, one can still obtain quite accurate results with Eq. (14). This is because the single-electron wave functions are not very sensitive to such a magnetic field (they are the same in linear order in the vector potential A, see Ref. 26).

\section{RESULTS AND DISCUSSION}

\section{A. Accuracy of Anderson's solution}

In Fig. 1 the zero-temperature order parameter is plotted versus $\rho$ as calculated from a numerical procedure based on Eq. (8) (solid curve) and Anderson's approximation (open squares). Two particular sets of $R$ and $d$ are chosen: for $R$ $=7 \mathrm{~nm}$ we subsequently consider (a) $d=3.17 \mathrm{~nm}$ and (b) $d$ $=3.76 \mathrm{~nm}$. Each set represents a superconducting resonance and so $\Delta(\rho)$ is enhanced as compared to the bulk zerotemperature gap $\Delta_{\text {bulk }}=0.25 \mathrm{meV}$. As seen, both solutions give practically the same results (differences are found to be less than $0.1 \%$ ). For $R=7 \mathrm{~nm}$ the centrifugal term in Eq. (9) almost does not change with $m$ on the energy scale controlled by $\hbar \omega_{D}$, i.e., $\frac{\hbar^{2}}{2 m_{e}} \frac{1}{\rho^{2}} \lesssim \frac{\hbar^{2}}{2 m_{e}} \frac{1}{R^{2}} \approx 1 \mathrm{meV} \ll \hbar \omega_{D}$. So, the single-electron states with different azimuthal quantum numbers are nearly degenerate. In particular, for $R=7 \mathrm{~nm}$ and $d=3.17 \mathrm{~nm}$ the lower edges of the single-electron subbands with $j=4$ and $|m|=0-13$ are located in the Debye window. This bundle of subbands makes major contribution to $\Delta(\rho)$ and other basic superconducting characteristics at this resonant point due to an enhanced density of states. At $d$ $=3.76 \mathrm{~nm}$ the bottoms of the subbands with $j=5$ and $|m|$ $=0-14$ are positioned in the vicinity of $E_{F}$, which results in the resonance illustrated by Fig. 1(b). The number of local maxima of $\Delta(\rho)$ is equal to $j+1$ and, so, increases by one
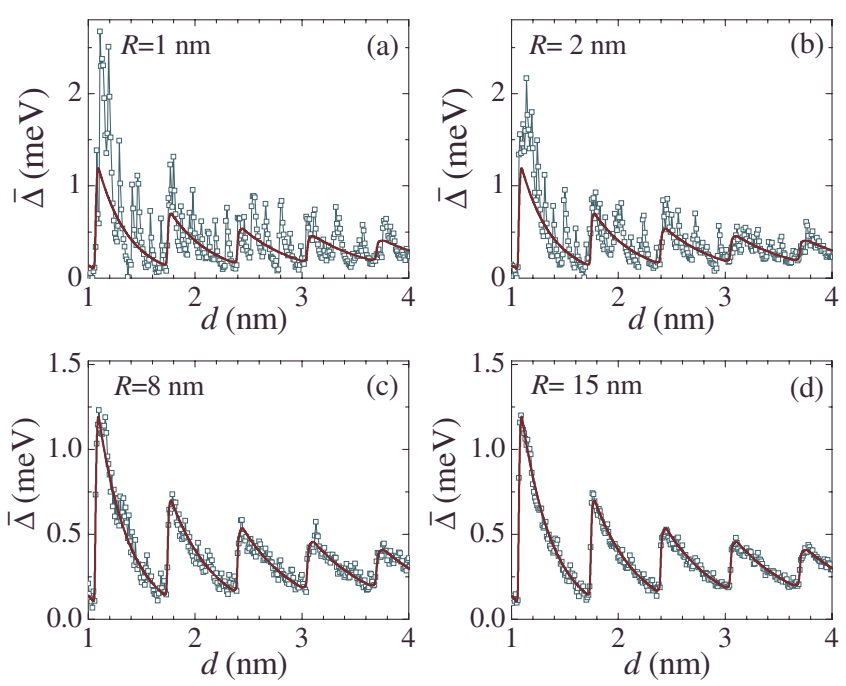

FIG. 2. (Color online) Crossover from an irregular to regular pattern of quantum-size oscillations: spatially averaged order parameter $\bar{\Delta}$ as function of $d$ for inner radii $R=1,2,8$, and $15 \mathrm{~nm}$.

when passing from panel (a) to panel (b). The contribution of a single-electron subband to the order parameter is given by $\Sigma_{k} u_{j m k}(\rho) v_{j m k}^{*}(\rho)\left(1-2 f_{j m k}\right) \approx \psi_{j m}^{2}(\rho) \Sigma_{k} U_{j m k} V_{j m k}^{*}\left(1-2 f_{j m k}\right)$, which is an oscillating function of $\rho$ with $j+1$ local maxima. Note that $j$ is the number of nodes of $\psi_{j m}(\rho)$ between $\rho=R$ and $\rho=R+d$ so that $\psi_{j m}^{2}(\rho)$ has $j+1$ local maxima. Therefore, when a superconducting resonance is governed by a bundle of nearly degenerate subbands with the same radial quantum number $j$, their input results in almost regular oscillations of $\Delta(\rho)$ as illustrated in Fig. 1. We remark that there are also superconducting resonances controlled by the subbands with different radial quantum numbers. In addition, when $R \rightarrow 0$ there are many examples of superconducting resonances controlled by simply one or two subbands. In such cases the order-parameter profile does not show regular oscillations. Its spatial distribution is very nonuniform and close to that of the pair condensate in a cylindrical nanowire (see, e.g., Ref. 33). Any changes in the profile of the order parameter and its spatially averaged value do not have any effect on the accuracy of Anderson's solution: our numerical investigations for $R=0-15 \mathrm{~nm}$ and $d=0-10 \mathrm{~nm}$ shows negligible deviations (within $0.1-0.3 \%$ ) of the results of Eq. (14) from the full $\mathrm{BdG}$ as data as based on the procedure of Eq. (8).

Thus, to abandon time-consuming numerical investigations that are based on the expansion given by Eq. (8), we will use Anderson's recipe of an approximate semianalytical solution to the BdG equations. As discussed above, corrections to this approximation are insignificant for $R$ $=0-15 \mathrm{~nm}$ and $d=0-10 \mathrm{~nm}$. For larger values of $d$ one can expect more pronounced deviations of Anderson's solution. ${ }^{36}$

\section{B. Quantum-size oscillations}

Quantization of the transverse electron motion results in superconducting quantum-size oscillations, i.e., oscillations of the basic superconducting quantities when changing in the geometrical parameters of the sample. Figure 2 shows how 
the spatially averaged superconducting order parameter $\bar{\Delta}$, i.e.,

$$
\bar{\Delta}=\frac{2}{d(2 R+d)} \int_{R}^{R+d} d \rho \rho \Delta(\rho),
$$

for different values of $R$ at $T=0$ (open squares). The same result for a superconducting nanofilm is shown by the solid curve. Panel (a) illustrates such thickness-dependent variations for $R=1.0 \mathrm{~nm}$. As seen, the two sets of numerical results differ significantly from one another. It is known $n^{23,37}$ that the thickness-dependent resonant enhancements in superconducting nanofilms appear almost regularly with period $\Delta d=\lambda_{F} / 2$. For small $d$ this period systematically decreases by about a few percent due to the shift-up of $E_{F}$ when decreasing $d$. The reason for such a regular appearance is that the single-electron spectrum is proportional to $\nu^{2} / d^{2}$ with $\nu$ the relevant quantum number controlling the transverse electron motion in nanofilms. This picture is different for nanowires. Here the quantum-size oscillations of superconducting properties are irregular. ${ }^{24}$ The difference in width between two neighboring resonant enhancements strongly fluctuates along with their magnitudes. Note that the hollow superconducting nanocylinder for $R=1.0 \mathrm{~nm}$ and $d=1-4 \mathrm{~nm}$ exhibits rather nanowirelike irregular oscillations of $\bar{\Delta}$ that are superimposed on top of the oscillations of $\bar{\Delta}$ in a corresponding nanofilm. From Fig. 2 we notice that with increasing $R$, the quantum-size oscillations in $\bar{\Delta}$ of a hollow nanocylinder approach more closely those of the corresponding nanofilm. However, the resonant enhancements over the bulk zerotemperature energy gap $\Delta_{\text {bulk }}=0.25 \mathrm{~nm}$ are still by a factor of two larger for the hollow nanocylinder. The centrifugal term $\frac{\hbar^{2}}{2 m} \frac{m^{2}}{\rho^{2}}$ in Eq. (9) is still of importance here: for $\rho=R$ we have $\frac{\hbar^{2}}{2 m_{e}} \frac{1}{R^{2}} \sim 9.52 \mathrm{meV}$, which is not negligible as compared to the Debye energy $\hbar \omega_{D}=32.31 \mathrm{meV}$. Therefore, the single-electron subbands with different $|m|$ produce well distinguished resonant enhancements of $\bar{\Delta}$ and this is the reason for the irregular variations in $\bar{\Delta}$ shown for the hollow nanocylinder in Figs. 2(a) and 2(b). For $R \geq 7-9 \mathrm{~nm}$ the centrifugal term in Eq. (9) plays a decreasing role and the singleelectron subbands with different $|m|$ become almost degenerate. This results in the formation of bundles of subbands with the same radial quantum number $j$ and different $|m|$. When a bottom of such a bundle comes into the Debye window a superconducting resonance appears. While increasing $R$, the sequence of these resonances exhibits a more regular pattern and, finally, we arrive at the nanofilm regime of the quantum-size oscillations. As seen from Figs. 2(c) and 2(d), this regime is already well approached at $R=8$ and 10 $\mathrm{nm}$. However, the deviations are still about $20 \%$ in panel (c) and are reduced to about $10 \%$ in (d). Thus, changing the geometrical parameters $R$ and $d$ results in a qualitatively different design of the single-electron subbands. The irregular distribution of the transverse electron levels at small $R$ leads to irregular quantum-size oscillations of $\bar{\Delta}$ (and other basic superconducting quantities) with $d$ (irregular regime). For sufficiently large $R$ the formation of bundles of the subbands
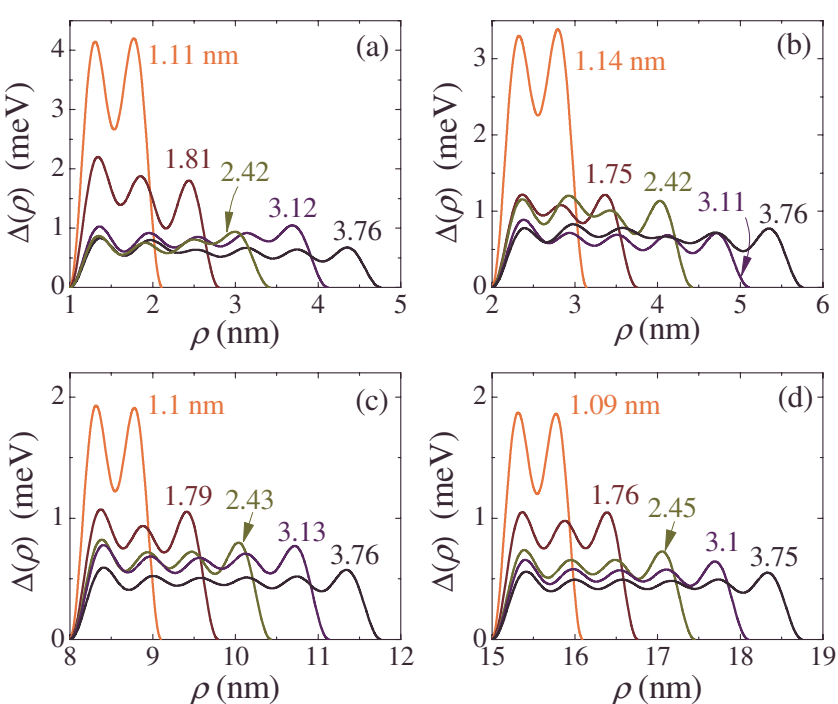

FIG. 3. (Color online) The radial distribution of the pair condensate in the hollow nanocylinder: (a) $R=1 \mathrm{~nm}$ and $d=1.11,1.81$, $2.42,3.12$, and $3.76 \mathrm{~nm} ; R=2 \mathrm{~nm}$ and $d=1.14,1.75,2.42,3.11$, and $3.76 \mathrm{~nm} ; R=8 \mathrm{~nm}$ and $d=1.1,1.79,2.43,3.13$, and $3.76 \mathrm{~nm}$; and $R=15 \mathrm{~nm}$ and $d=1.09,1.76,2.45,3.1$, and $3.75 \mathrm{~nm}$.

with the same radial quantum number $j$ but different $m$ makes the quantum-size oscillations regular, yielding almost equidistant superconducting enhancements (regular regime) at $d_{j}=1.09 \mathrm{~nm}, 1.79 \mathrm{~nm}, 2.42 \mathrm{~nm}, 3.1 \mathrm{~nm}$, and $3.75 \mathrm{~nm}$ for $j=1-5$, respectively. The reason for the observed crossover from the irregular pattern to the regular regime is the interplay of quantum confinement and sample geometry.

Figure 3 adds more information to the discussion of the previous paragraph. Here the radial profiles of the order parameter $\Delta(\rho)$ are plotted for a series of the superconducting resonances. Inner radii are taken the same as in Fig. 2. Thicknesses are chosen so that to take the most profound resonant enhancement in the vicinity of $d=d_{j}$ for $j=1-5$. (the concrete values of $d$ are given in Fig. 3). When comparing these profiles with the spatial distribution of the pair condensate in aluminum nanofilms at $d=d_{j},{ }^{37}$ one can find pronounced differences for the irregular regime illustrated in panels (a) and (b). First of all, enhancements of $\Delta(\rho)$ for the nanocylinder are more significant, which can also be seen from Figs. 2(a) and 2(b). Second, nanofilms are specified by the symmetric order-parameter distribution, i.e., $\left.\Delta(\rho)\right|_{\rho=R+x}=\left.\Delta(\rho)\right|_{\rho=R+d-x}$. Such a symmetry does not generally hold for the hollow nanocylinder. This is clearly seen in panels (a) and (b). As for Figs. 3(c) and 3(d), we arrive at the regular regime and there are no significant deviations from the filmlike spatial distribution of the superconducting condensate. As already mentioned in Sec. III A, here the number of local maxima of $\Delta(\rho)$ is equal to $j+1$, where $j$ is the radial quantum number related to the bundle of the subbands governing the corresponding superconducting resonance in the regular regime. The distance between two neighboring maxima is close to $\lambda_{F} / 2$ with $\lambda_{F}=2 \pi \hbar /\left(2 m_{e} E_{F}\right)^{1 / 2}$ the three-dimensional (3D) Fermi wavelength for our effective Fermi level. Note that a superconducting resonance appears exactly at the point where the relevant bundle of single-electron subbands 
touches the Fermi surface. As the number of the local maxima in $\Delta(\rho)$ increases by one from a resonant to the next one, we can expect that the difference in $d$ for two neighboring resonances is also $\lambda_{F} / 2$. Recall that for $d=1-3 \mathrm{~nm} E_{F}$ is systematically shifted up from $0.9 \mathrm{eV}$ with a decrease in $d$ (see the discussion in the last paragraph of Sec. II A). Dependence of $E_{F}$ on $R$ is almost negligible.

Notice that, as seen from Eq. (19), the resonant enhancements of superconducting properties depend on the interaction matrix elements $J_{j^{\prime} m^{\prime}, j m}$. So, a minor disorder, e.g., due to disordered interface between the sample and substrate or due to surface roughness, is expected to smoothen the quantum-size oscillations through the above matrix elements. ${ }^{38}$ Another point is that such a disorder can also influence the mean density of single-electron states in the Debye window through a broadening of the transverse electron levels. In particular, this can somewhat shift superconducting resonances, violating the regular pattern at large $R$. The broadening should exceed the intersubband spacing $\delta_{\text {sub }}$ in order to completely destroy the formation of distinguished single-electron subbands. Note that tunneling experiments with single-crystalline $\mathrm{Pb}$ nanofilms demonstrate clear signatures of the formation of well distinguished transverse electron levels. ${ }^{4,6,9}$

\section{Andreev-type states induced by radial quantum confinement}

As seen from Figs. 1-3, the formation of multiple singleelectron subbands results in significant modifications of the spatial distribution of the superconducting condensate and in quantum-size oscillations (both regular and strongly irregular) of basic superconducting properties. Another interesting consequence of the appearance of multiple subbands is the multigap structure of the quasiparticle excitations. As follows from Eq. (16), each single-electron subband is specified by $\Delta_{j m}$, the energy gap of excitations in a given subband. Based on Eqs. (6), (14), and (16), this quantity can be represented as

$$
\Delta_{j m}=\int d^{3} r\left[u_{j m k}^{*}(\mathbf{r}) \Delta(\mathbf{r}) u_{j m k}(\mathbf{r})+v_{j m k}^{*}(\mathbf{r}) \Delta(\mathbf{r}) v_{j m k}(\mathbf{r})\right],
$$

i.e., $\Delta_{j m}$ is the average value of the order parameter as "seen" by quasiparticles in the corresponding single-electron subband. If the order parameter is uniform (e.g., in bulk), $\Delta_{j m}$ does not depend on the relevant quantum numbers because of the normalization condition, ${ }^{32}$ i.e., $\int d^{3} r\left[\left|u_{j m k}(\mathbf{r})\right|^{2}\right.$ $\left.+\left|v_{j m k}(\mathbf{r})\right|^{2}\right]=1$. In the presence of quantum confinement the superconducting order parameter is always position dependent due to a broken translational symmetry and, so, we obtain energy gaps sensitive to the quantum numbers, i.e., the multigap case. From Eq. (23) it follows that when quasiparticles are successive in avoiding local enhancements in $\Delta(\mathbf{r})$, they are specified by smaller $\Delta_{j m}$ 's and, so, by generally lower energies: to some extent a spatial variation in the superconducting order parameter appears to be analogous to a potential well for quasiparticles. This is the basis of the
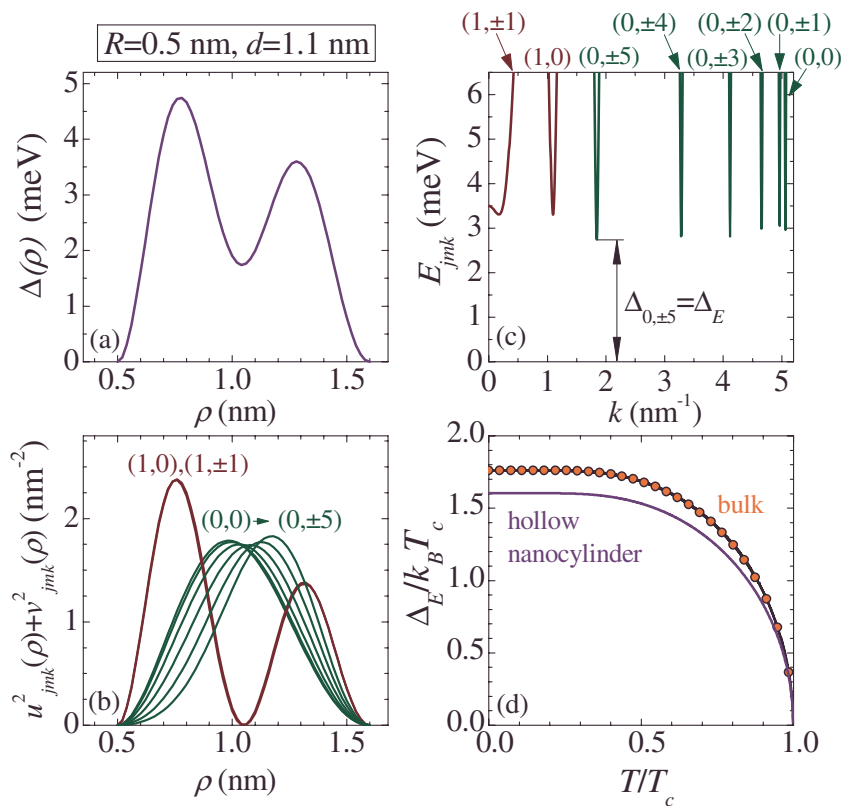

FIG. 4. (Color online) The hollow nanocylinder for $R=0.5 \mathrm{~nm}$ and $d=1.1 \mathrm{~nm}$ : (a) the radial distribution of the pair condensate; (b) the quasiparticle energy $E_{j m k}$ versus $k>0$ for relevant singleelectron subbands $(j, m)=(0,0)-(0, \pm 5)$ and $(1,0),(1, \pm 1)$; (c) the radial distributions of the corresponding quasiparticles; and (d) the ratio $\frac{\Delta_{E}}{k_{B} T_{c}}$ as function of $T$ for the hollow cylinder (solid curve) and bulk (circles).

well-known Andreev reflection (see, e.g., Refs. 25 and 39) which we refer to as Andreev mechanism. Its interplay with quantum confinement is illustrated by Fig. 4 for $R=0.5 \mathrm{~nm}$ and $d=1.1 \mathrm{~nm}$ (the point of a superconducting resonance). Here panels (a) and (b) show the order parameter and the radial distribution of quasiparticles $u_{j m k}^{2}(\rho)+v_{j m k}^{2}(\rho)=\psi_{j m}^{2}(\rho)$ (the latter does not depend on $k$ in Anderson's approximation). There are eight relevant subbands making a contribution to the superconducting characteristics at the chosen geometrical parameters: $(j, m)=(0,0)-(0, \pm 5)$ and $(1,0)-(1, \pm 1)$. The bottom of the single-electron subband with $(j, m)=(1, \pm 1)$ is situated in the vicinity of the Fermi surface and, so, it makes a major contribution to $\Delta(\rho)$ due to an enhanced density of states. This is the subband that controls the resonant enhancement at $R=0.5 \mathrm{~nm}$ and $d$ $=1.1 \mathrm{~nm}$ (resonant subband) and determines the corresponding radial profile of the order parameter with the two local maxima [compare with the radial distribution of quasiparticles $(1, \pm 1)$ in panel (b)]. Other subbands play a less important role at this superconducting resonance. The most significant contribution among them is due to the states with $(j, m)=(1,0)$. As these states have practically the same radial distribution as those with $(j, m)=(1, \pm 1)$ [the curves for $(1, \pm 1)$ and (1,0) in Fig. 4(b) are almost indistinguishable), then, based on Eq. (23), one can expect that $\Delta_{1,0}$ should be nearly the same as $\Delta_{1, \pm 1}$. This expectation agrees with our numerical results given in Fig. 4(c). Here the quasiparticle energy $E_{j m k}$ is plotted versus the positive values of $k$ and, as seen, each single-electron subband is specified by its own quasiparticle branch whose minimum energy gives the subband superconducting gap. 

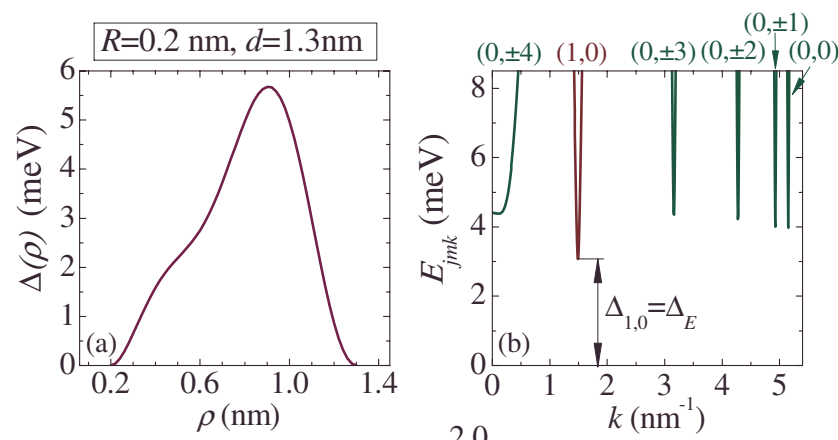

$(0, \pm 1)$

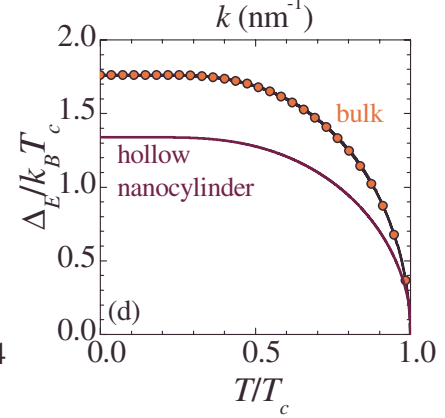

FIG. 5. (Color online) The same as in the previous figure but for $R=0.2 \mathrm{~nm}$ and $d=1.2 \mathrm{~nm}$ ).

By virtue of the definition of $j$ (the number of nodes in the radial direction), the spatial distribution of quasiparticles with $j=0$ is characterized by one maximum [see panel (b)] located next to the point of a local minimum of $\Delta(\rho)$ at $\rho$ $\approx 1.05 \mathrm{~nm}$. Therefore, $\Delta_{j m}$ is generally lower for $j=0$ [see Fig. 4(c)] and, so, quasiparticles with $(j, m)=(0,0)-(0, \pm 5)$ can be interpreted as Andreev-type states induced by the radial quantum confinement. The total energy gap in the quasiparticle spectrum $\Delta_{E}$ is determined by the lowest subbanddependent gap, i.e., $\Delta_{E}=\Delta_{0, \pm 5}$, see panel (c). Due to a relatively small density of states, such Andreev-type quasiparticles produce only a minor contribution to the basic superconducting characteristics, e.g., the critical temperature. However, they control the low-lying energy excitations in a superconductor. This is illustrated by Fig. 4(d), where the ratio of $\Delta_{E}$ to $T_{c}$ is shown as function of temperature. Subband $(0, \pm 5)$ has the lowest energy gap and determines $\Delta_{E}$ but does not make a significant contribution to $T_{c}$. The latter is controlled by the resonant subband $(1, \pm 1)$ with the higher-energy gap. This is why the ratio $\Delta_{E} / k_{B} T_{c}$ is decreased as compared to bulk. As seen from panel (d), such a decrease is about $10 \%$. This is by a factor of 2 smaller than the reduction in $\Delta_{E} / k_{B} T_{c}$ due to similar Andreev-type states in a cylindrical nanowire with radii $1-2 \mathrm{~nm} .{ }^{25}$ The point is that the superconducting condensate in a hollow nanocylinder exhibits generally more uniform spatial patterns, which results in a less profound difference between subbanddependent gaps. The centrifugal energy appears to be a significant contributor to a nonuniform distribution of the pair condensate and the role of this term is reduced due to the annulus in a hollow nanocylinder. This is the reason why the Andreev-type states play a more important role with decreasing $R$. This is seen from Fig. 5 that shows the same data as in Fig. 4 but for $R=0.2 \mathrm{~nm}$ and $d=1.1 \mathrm{~nm}$, i.e., for nearly the same thickness as previously but for a significantly smaller inner radius. The superconducting resonance illustrated by Fig. 5 is governed by the single-electron subband with $(j, m)=(0, \pm 4)$ and, as seen, the maximum of $\Delta(\rho)$ is located at the same point as the maximum of the radial quasiparticle distribution for $(j, m)=(0, \pm 4)$. In addition to states $(0, \pm 4)$, there are five more single-electron subbands making a minor contribution to the superconducting characteristics. Four of them are specified by $j=0$ and, so, spatial distributions of the corresponding quasiparticles are close to that of the resonant subband. So, there is a minor difference between $\Delta_{j m}$ 's with $j=0$. However, it is clearly seen that $\Delta_{j m}$ (with $j=0$ ) is slightly diminished when decreasing $|m|$. The reason is that the maximum of the quasiparticle distribution for the states with $j=0$ is shifted to smaller $\rho$ with decreasing $|m|$. It means that the value of the corresponding integral in Eq. (23) is reduced. One more relevant single-electron subband is $(j, m)=(1,0)$. The radial distribution of the quasiparticles in this subband has two local maxima and the most significant of them is located far to the left from the maximum of $\Delta(\rho)$. As a result, we obtain a significant drop of $\Delta_{1,0}$ as compared to the energy gap of the resonant subband, i.e., $\Delta_{0, \pm 4}$. Such a drop leads to a significant decrease (now it is about $20 \%$ ) in the ratio $\Delta_{E} / k_{B} T_{c}$, where $\Delta_{E}=\Delta_{1,0}$ and $T_{c}$ is controlled by the states with $(j, m)=(0, \pm 4)$ (notice the reasonable estimate $\left.k_{B} T_{c} \approx 1.76 \Delta_{0, \pm 4}\right)$.

For nonresonant sets of $R$ and $d$, when the bottoms of all relevant subbands are far below the Fermi level, the order parameter (and $T_{c}$ ) is not enhanced as compared to bulk and exhibits, as a rule, a more uniform spatial distribution. In this case the Andreev mechanism plays a less important role and the ratio $\Delta_{E} / k_{B} T_{c}$ is close to its bulk value. However, deviations from bulk can still be significant, see Ref. 25 and, so, this effect of Andreev-type states induced by quantum confinement can be observed even in the presence of fluctuations of the transverse dimensions $R$ and $d$ when all the results should be averaged over relevant intervals of their variations. Interplay of the Andreev mechanism and quantum confinement in nanoscale superconductors can be probed experimentally by means of simultaneous measurements of $T_{c}$ and $\Delta_{E}$. So, it is interesting to have an idea about how the quasiparticle density of states measured in tunneling experiments is modified by quantum confinement. The dependence of this density of states on the quasiparticle energy $E$ (in units of $\Delta_{\text {bulk }}$ ) is illustrated for a hollow nanocylinder by Fig. 6 for: (a) $R=0.5 \mathrm{~nm}$ and $d=1.1 \mathrm{~nm}$; (b) $R=2.0 \mathrm{~nm}$ and $d$ $=1.1 \mathrm{~nm}$; and (c) $R=1.0 \mathrm{~nm}$ and $d=1.77 \mathrm{~nm}$ (all sets are resonant points). In addition, panel (d) shows the quasiparticle energy $E_{j m k}$ versus $k>0$ for the latter set of $R$ and $d$. Multiple peaks (more precisely, multiple square-root type of divergences) in the quasiparticle density of states [in units of $N(0)$, the bulk normal density of states] reflects the formation of the multigap structure. In particular, in panel (a) we can see a significant peak at $E \approx 13.2 \Delta_{\text {bulk }}$ and two less important peaks at $E / \Delta_{\text {bulk }}=11$ and 11.5 . The major peak is due to the contribution of quasiparticles from the resonant singleelectron subband with $(j, m)=(1, \pm 1)$ [see Fig. 4(c)]. Two small peaks shifted towards smaller energies are due to Andreev-type states induced by the radial quantum confinement, i.e., $E=11.5 \Delta_{\text {bulk }}$ comes from $(j, m)=(1,0)$ and $E$ $=11 \Delta_{\text {bulk }}$ is from $(j, m)=(0, \pm 3)-(0, \pm 5)$. There are addi- 

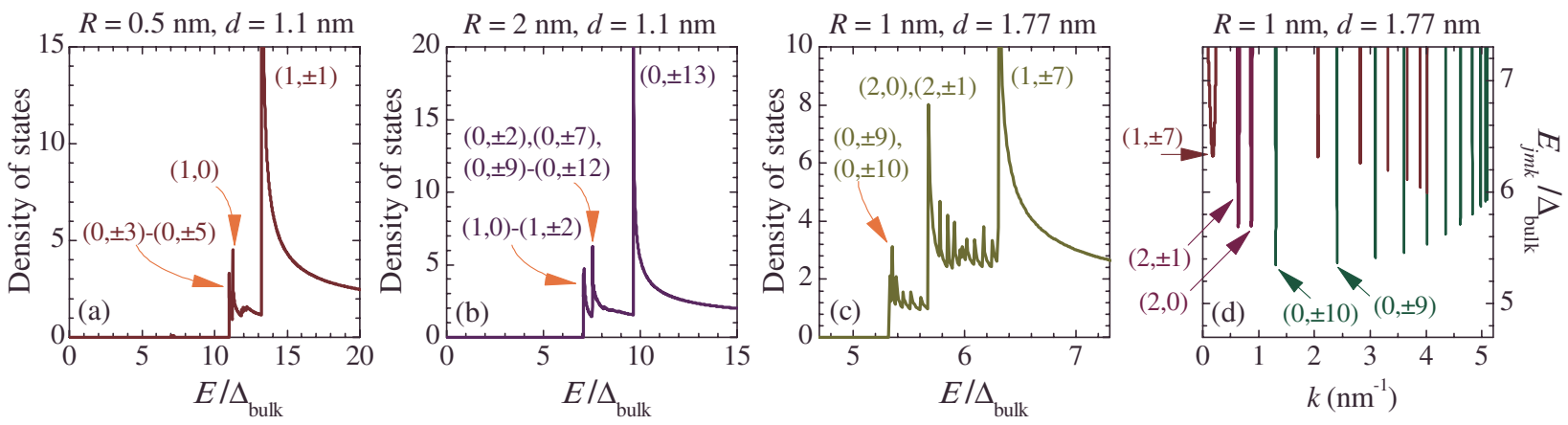

FIG. 6. (Color online) The quasiparticle density of states in the irregular regime: (a) for $R=0.5 \mathrm{~nm}$ and $d=1.1 \mathrm{~nm}$; (b) for $R$ $=2.0 \mathrm{~nm}$ and $d=1.1 \mathrm{~nm}$; and (c) for $R=1.0 \mathrm{~nm}$ and $d=1.77 \mathrm{~nm}$. Panel (d) shows $E_{j m k}$ as dependent on $k$ for the same parameters as in (c).

tional divergences in the quasiparticle density of states at $E$ $=12-13 \mathrm{meV}$. They are associated with the single-electron subbands $(0,0)-(0, \pm 2)$. The corresponding peaks in panel (a) are thin and practically invisible.

A similar picture occurs for $R=2.0 \mathrm{~nm}$ and $d=1.1 \mathrm{~nm}$. As seen from Fig. 6(b), we again obtain one major peak due to the resonant subband labeled now by $(j, m)=(0, \pm 13)$ (at $\left.E / \Delta_{\text {bulk }}=9.6\right)$. In addition, there are two less pronounced peaks: at $E / \Delta_{\text {bulk }}=7$ due to the subbands $(1,0)-(1, \pm 2)$ and at $E / \Delta_{\text {bulk }}=7.6$ from subbands $(0, \pm 2),(0, \pm 7)$ and $(0, \pm 9)-(0, \pm 12)$. Peaks from other subbands almost disappear.

For more specific information, Fig. 6(c) shows all peaks in the quasiparticle density of states, both significant and secondary in importance, at a more detailed energy scale for $R=1.0 \mathrm{~nm}$ and $d=1.77 \mathrm{~nm}$. For convenience, panel (d) demonstrates $E_{j m k}$ as function of $k$ for the same parameters. These parameters correspond to a superconducting resonance induced by the single-electron subband with $(j, m)$ $=(1, \pm 7)$ that makes a major contribution to the basic superconducting quantities and is specified by the subband gap $\Delta_{1, \pm 7}=6.35 \Delta_{\text {bulk }}$ [see panel (d)]. As a result, we have a profound major peak at $E / \Delta_{\text {bulk }}=6.3$ in Fig. 6(c). It is seen from Fig. 6(d) that there is also a subband with $(j, m)=(1, \pm 6)$ which is characterized by nearly the same superconducting gap, i.e., $\Delta_{1, \pm 6} \approx \Delta_{1, \pm 7}$. However, its contribution to the density of states is much less significant. The second important peak is situated at $E / \Delta_{\text {bulk }}=5.7$, which is the energy gap for subbands $(2,0)$ and $(2, \pm 1): \Delta_{2,0}=\Delta_{2, \pm 1}=5.7 \Delta_{\text {bulk }}$. The third pronounced peak in Fig. 6(c) forms due to two divergences in the density of states due to the subbands $(0, \pm 9)$ and $(0, \pm 10)$. Here $\Delta_{0, \pm 9} \approx \Delta_{0, \pm 10}=5.3 \Delta_{\text {bulk }}$, see Fig. 6(d). In addition, one can see clear signatures of less important divergences in Fig. 6(c). In particular, between the two significant peaks located at $E / \Delta_{\text {bulk }}=5.7$ and $E / \Delta_{\text {bulk }}=6.3$, there are a series of peaks coming from the subbands with $(j, m)$ $=(1,0)-(1, \pm 6)$ (the larger the absolute value of the azimuthal quantum number the smaller the corresponding subband energy gap). In turn, the subbands $(0,0)-(0, \pm 8)$ are responsible for a series of peaks in the energy domain $E / \Delta_{\text {bulk }}=5.3-5.7$.

As mentioned above, the formation of Andreev-type states induced by quantum confinement is related to a spatially nonuniform distribution of the pair condensate. In the regime of irregular quantum-size oscillations (see Sec. III B), i.e., for sufficiently small $R$, the order parameter exhibits pronounced spatial variations, which results in a significant role of the Andreev mechanism. When increasing $R$, we pass to the regime of regular quantum-size oscillations because single-electron subbands with different absolute values of the azimuthal quantum number are almost degenerate for large enough $R$ and form bundles. In this case the spatial variations in $\Delta(\rho)$ become less and less pronounced and, as a result, the multigap structure disappears. In particular, Figs. 7(a) and 7(b) show the quasiparticle density of states and the quasiparticle energy as function of $k$ for $R=15 \mathrm{~nm}$ and $d$ $=1.09 \mathrm{~nm}$. This is the point of a superconducting resonance induced by a bundle of single-electron subbands with $j=1$. The states with $(j, m)=(1,0)-(1, \pm 14)$ makes a contribution to the superconducting characteristics of about $60 \%$ here. The rest is due to the subbands $(0,0)-(0, \pm 79)$. As seen from Fig. 7(b), the quasiparticle branches with $(j, m)$ $=(0, \pm 7)$ are almost degenerate. However, branches with $j$ $=1,|m|>7$ are still well distinguished. Andreev mechanism is of no importance any more and, so, the subband energy gaps do practically not depend on the quantum numbers: $\Delta_{j m}=6 \Delta_{\text {bulk }}$ for $(j, m)=(1,0)-(1, \pm 14)$ and $(0,0)-(0, \pm 78)$. Therefore, we can see a profound peak in the quasiparticle density of states at $E / \Delta_{\text {bulk }}=6$. There are two additional subbands $(0, \pm 79)$ and $(1, \pm 15)$ that contribute to the superconducting quantities. As follows from Fig. 7(b), the spectroscopic gaps for these states are $13.5 \Delta_{\text {bulk }}$ and $18 \Delta_{\text {bulk }}$ with the corresponding peaks in Fig. 7(a). These spectroscopic gaps are not directly related to $\Delta_{0, \pm 79}$ and $\Delta_{1, \pm 15}$ because the bottoms of both subbands are located above the Fermi level (but in the Debye window). It means that $\xi_{j m k}$ is different from zero at the point of the minimum of the corresponding quasiparticle energy $E_{j m k}$ taken as function of $k$ [see Eq. (16)]. Hence, the above spectroscopic gaps are shifted up by a nonzero single-electron energy. It is interesting that $\Delta_{0, \pm 79}$ and $\Delta_{1, \pm 15}$ are smaller than $6 \Delta_{\text {bulk. }}$. However, this does not have any effect on the ratio of $\Delta_{E}$ to $k_{B} T_{c}$ : these subbands produce a negligible contribution to the superconducting characteristics due to the large corresponding spectroscopic gaps. In Fig. 7(c) one can see another example of the energy dependence of the quasiparticle density of states. Thus, as seen, the multigap structure is almost degenerate in the regular regime and Andreev-type states do not play such essential role as for smaller inner radii, i.e., in the case of irregular quantum-size oscillations. 

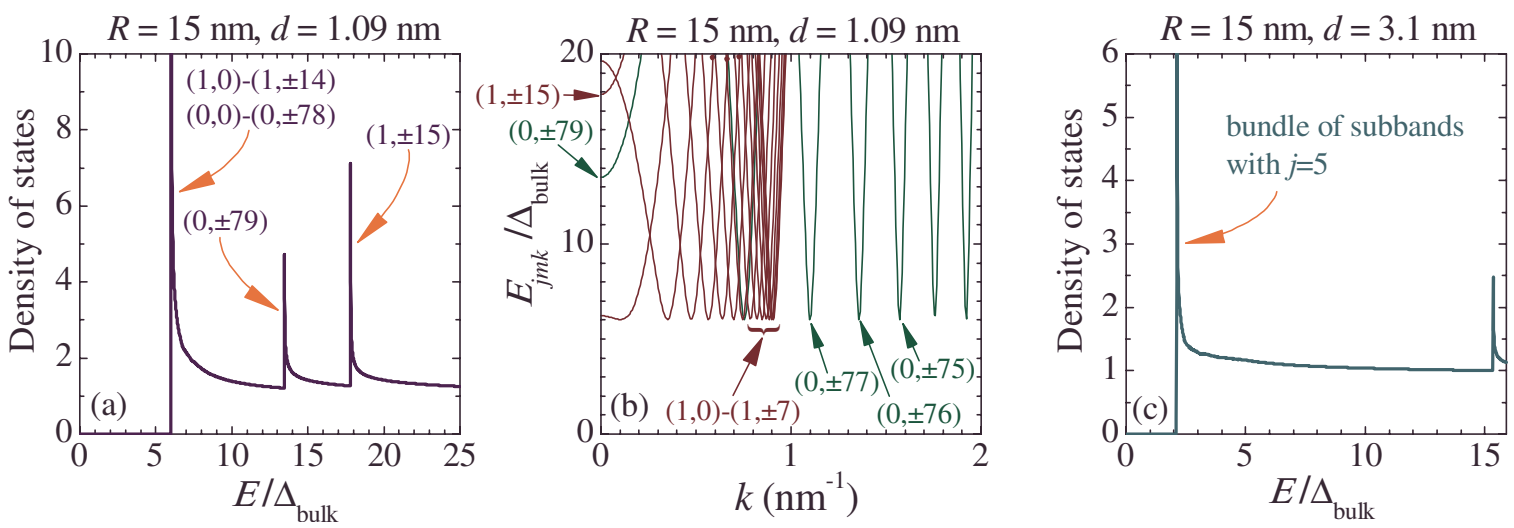

FIG. 7. (Color online) Hollow nanocylinder in the regular regime: (a) the quasiparticle density of states versus the energy $E$ at $R$ $=15 \mathrm{~nm}$ and $d=1.09 \mathrm{~nm}$; (b) $E_{j m k}$ as function of $k$ (in the interval $k=0-2 \mathrm{~nm}^{-1}$ ) for the relevant single-electron subbands with $(j, m)$ $=(0,0)-(0, \pm 79)$ and $(1,0)-(1, \pm 15)$; and (c) the density of states versus the quasiparticle energy $E$ for $R=15 \mathrm{~nm}$ and $d=3.1 \mathrm{~nm}$.

Concluding this section, we note that the radial profile of the superconducting order parameter is mainly determined by single-electron subbands with the bottoms close to the Fermi surface. These subbands are characterized by slow motion of electrons in the direction parallel to the nanocylinder (here the major part of the corresponding singleelectron energy comes from the radial and azimuthal degrees of freedom). In particular, typical longitudinal wavelengths for electrons in a resonant subband are about $20 \mathrm{~nm} \gg \lambda_{F}$. Propagation of such long waves is not sensitive to the surface imperfections with the characteristic size of the order of the metallic unit-cell dimensions. Thus, the profile of the order parameter and, so, the formation of Andreev states induced by quantum confinement will be stable against surface roughness.

\section{CONCLUSIONS AND DISCUSSIONS}

By numerically solving the BdG equations, we have investigated how quantum confinement modifies superconductivity in a hollow metallic nanocylinder. The radial quantization of the electron motion results in a splitting of the conduction band into a series of subbands whose lower edges move in energy when changing the inner radius $R$ and thickness $d$. Such a multisubband structure leads to pronounced quantum-size superconducting oscillations, e.g., the superconducting properties varies with $d$ at a fixed $R$. We have found that the character of these variations changes qualitatively with an increase in $R$ : the irregular pattern is replaced by almost equidistant superconducting resonant enhancements. The reason for such a crossover is the change in the role of the centrifugal energy. When $R$ increases, this energy is diminished so that single-electron subbands with different absolute values of the azimuthal quantum number $m$ (but with the same radial quantum number $j$ ) are nearly degenerate in energy. As a result, they form bundles of subbands responsible for equidistant resonant enhancements in the basic superconducting quantities as functions of $d$. We have shown that the formation of subbands can result in multiple superconducting gaps due to the interplay between quantum confinement and the Andreev mechanism. The difference be- tween the subband-dependent superconducting gaps is significant in the irregular regime but they are almost equal when coming to the regular side of the crossover. Here the ratio of the critical temperature to the energy gap approaches its bulk value while it is reduced by $20-30 \%$ due to Andreev-type states in the irregular regime. The role of Andreev-type states induced by quantum confinement is significant for small inner radii but becomes minor in the regular domain.

We would like to remark that fluctuations are known to play a more serious role for lower dimensionality. So, one can expect deviations from the mean-field results for nanoscale superconducting systems. However, a recent experimental study of $\mathrm{Pb}$ superconducting single-crystalline nanofilms showed a good agreement with the mean-field results even for film thicknesses down to 2-5 monolayers (0.5-1.0 $\mathrm{nm}){ }^{9}$ For superconducting nanowires it is believed ${ }^{16}$ that quantum-phase fluctuations suppress the superconducting state for diameters below 5-8 $\mathrm{nm}$. This expectation is in agreement with the experimental results of Ref. 14, where a crossover from the superconducting to normal state was reported for an aluminum nanowire with a diameter of about 8 $\mathrm{nm}$. However, results of another group ${ }^{15}$ demonstrate no signature of such a crossover in aluminum nanowires with diameters 5-6 nm. Thus, based on the above experimental data, we may expect that the mean-field approach is reasonable for hollow nanocylinder when $d \geqslant 1 \mathrm{~nm}$ and $R+d$ 2-3 nm, with $R+d$ the total radius of the hollow cylinder. In addition, we remark that the existing treatment of superconducting fluctuations is questionable in the presence of multiple single-electron subbands in nanowires/nanofilms. Due to scattering of electrons between different subbands, this has nothing to do with the one-dimensional/twodimensional limit and, so, the superconducting fluctuations, both quantum and thermal, will be different. Thus, one can expect that when the number of the relevant single-electron subbands is significantly larger than one, the 3D case is being approached and the mean-field approximation is justified. However, even when only a small number of subbands is occupied, the mean-field approximation can still yield reasonable results like in $\mathrm{Pb}$ and In single-crystalline oneatomic-layer thick films ${ }^{40}$ with only one relevant singleelectron subband. 
We also note that some smearing of the spatial profile of the order parameter can be expected when including the nonlocal-gap operator in the $\mathrm{BdG}$ equations. More specifically, using a nonlocal-gap representation of the BdG equations, one simply avoids the delta-function approximation of the pair interaction in favor of a more detailed potential. Then, when solving Anderson's BCS-type equation [see Eq. (19)], the only difference is that new interaction-matrix elements $J_{j^{\prime} m^{\prime}, j m}$ will appear in the problem. While resonant enhancements can be sensitive to the interaction-matrix elements, this is not the case for the radial profile of the order parameter that is governed by quantum confinement. Indeed, the main contribution to the order parameter at a resonant point comes from the single-electron subband whose bottom is in the vicinity of the Fermi surface. It means that, by virtue of Eq. (14), the radial profile of the order parameter is determined by the radial single-electron wave function associated with the above subband. Hence, the spatial profile of the order parameter will change only if the relevant singleelectron wave functions will be different. However, such a difference will not be pronounced because the electronelectron interaction itself has almost no effect on the wave functions in superconducting nanowires/nanofilms. From the numerical results of Ref. 41, this effect is estimated to be within a few percent. In other words, the order parameter is directly related to the Cooper-pair wave function and a wave function of two particles in a quantum-confining geometry is much more sensitive to tight confinement than to details of the interparticle interaction. Generally, the strength of quantum confinement in nanowires/nanofilms can be specified by the dimensionless parameter $\delta_{\text {sub }} / \Delta_{\text {bulk }}$ : when it is larger than one, quantum-confinement effects predominate. For nanowires $\delta_{\text {sub }} \sim \frac{\hbar^{2}}{2 m_{e}} \frac{\pi^{2}}{\mathrm{R}^{2}}$ with $\mathrm{R}$ the nanowire radius (we note that for a hollow nanocylinder $\mathrm{R}$ is a complicated function of $R$ and $d$ ). Typical values for the energy gap in bulk metallic superconductors are $\Delta_{\text {bulk }}=0.1-1.5 \mathrm{meV}$ (see, e.g., Ref. 16). This allows one to find that $\delta_{\text {sub }} / \Delta_{\text {bulk }}>1$ when $\mathbb{R}$ $<20-80 \mathrm{~nm}$. In particular, for aluminum this leads to $\mathbb{R}$ $<40 \mathrm{~nm}$.

It is also of interest to discuss the possible effect of surface imperfections on the superconducting resonances in quantum nanowires. Surface roughness can influence both the longitudinal and transverse motion of electrons. As discussed above, the basic characteristics of the system at the point of a superconducting resonance are controlled by the corresponding resonant single-electron subband whose bottom is situated in the vicinity of the Fermi level. In such a subband the main contribution to the single-electron energy comes from the transverse motion and, so, the longitudinal motion of electrons is slow. In particular, from Fig. 4 we learn that the resonant subband at $R=0.5 \mathrm{~nm}$ and $d$ $=1.1 \mathrm{~nm}$ is specified by $(j, m)=(1, \pm 1)$, and the corresponding subband-dependent longitudinal Fermi wave number is about $0.2-0.3 \mathrm{~nm}^{-1}$. Similar values for the longitudinal Fermi wave number in the resonant subband with $(j, m)=(0, \pm 4)$ can be found from Fig. 5 for $R=0.2 \mathrm{~nm}$ and $d=1.3 \mathrm{~nm}$. In general, the characteristic longitudinal wavelengths of electrons in a resonant subband are larger than 15-30 nm. Propagation of such long waves will not be sensitive to surface imperfections with characteristic sizes of about the dimensions of the metallic unit cell. Another possible effect of surface roughness is a broadening of the transverse electron levels and, consequently, a smearing of the lower edge of a single-electron subband. This will lead to an uncertainty in the intersubband energy spacing and, so, one can expect that when this uncertainty is close to or larger than the Debye energy $\hbar \omega_{D}$, an effect of such a smearing on quantum-size oscillations of basic superconducting properties can be pronounced. The uncertainty in the intersubband energy spacing for nanowires is estimated as $\Delta \delta_{\text {sub }}$ $=\frac{\pi^{2} \hbar^{2}}{m_{e} \mathbb{R}^{3}} \Delta \mathrm{R}$ with $\Delta \mathrm{R}$ the characteristic length of the radius fluctuations. Taking $\Delta \mathbb{R}=0.3 \mathrm{~nm}$ we can obtain that $\Delta \delta_{\text {sub }}$ $=30 \mathrm{meV}$ for $\mathbb{R}=2 \mathrm{~nm}$ whereas $\Delta \delta_{\text {sub }}=8 \mathrm{meV}$ for $\mathbb{R}$ $=3 \mathrm{~nm}$. The Debye energy for aluminum is about $32 \mathrm{meV}$, see discussion in the last paragraph of Sec. II A. Hence, smearing for quantum-size oscillations of the basic superconducting quantities due to surface roughness can be expected for aluminum nanowires with radii less than $2 \mathrm{~nm}$. Thus, in a hollow nanocylinder such a smearing can be of relevance for $R+d<2 \mathrm{~nm}$.

\section{ACKNOWLEDGMENTS}

This work was supported by the Flemish Science Foundation (FWO-VI), the Interuniversity Attraction Poles Programme, Belgian States, Belgian Science Policy (IAP) and the ESF-AQDJJ network.

\footnotetext{
*arkady.shanenko@ua.ac.be

${ }^{1}$ Y. Guo, Y. F. Zhang, X. Y. Bao, T. Z. Tang, L. X. Zhang, W. G. Zhu, E. G. Wang, Q. Niu, Z. Q. Qiu, J. F. Jia, Z. X. Zhao, and Q. K. Xue, Science 306, 1915 (2004).

${ }^{2}$ M. M. Özer, J. R. Thompson, and H. H. Weitering, Nat. Phys. 2, 173 (2006)

${ }^{3}$ M. M. Özer, J. R. Thompson, and H. H. Weitering, Phys. Rev. B 74, 235427 (2006).

${ }^{4}$ D. Eom, S. Qin, M. Y. Chou, and C. K. Shih, Phys. Rev. Lett. 96, 027005 (2006).

${ }^{5}$ T. Nishio, T. An, A. Nomura, K. Miyachi, T. Eguchi, H. Sakata,
}

S. Z. Lin, N. Hayashi, N. Nakai, M. Machida, and Y. Hasegawa, Phys. Rev. Lett. 101, 167001 (2008).

${ }^{6}$ K. Wang, X. Zhang, M. M. T. Loy, T.-C. Chiang, and X. Xiao, Phys. Rev. Lett. 102, 076801 (2009).

${ }^{7}$ T. Cren, D. Fokin, F. Debontridder, V. Dubost, and D. Roditchev, Phys. Rev. Lett. 102, 127005 (2009).

${ }^{8}$ C. Brun, I. Po Hong, F. Patthey, I. Y. Sklyadneva, R. Heid, P. M. Echenique, K. P. Bohnen, E. V. Chulkov, and W. D. Schneider, Phys. Rev. Lett. 102, 207002 (2009).

${ }^{9}$ S. Y. Qin, J. Kim, Q. Niu, and C. K. Shih, Science 324, 1314 (2009). 
${ }^{10}$ M. Tian, J. Wang, J. S. Kurtz, Y. Liu, M. H. W. Chan, T. S. Mayer, and T. E. Mallouk, Phys. Rev. B 71, 104521 (2005).

${ }^{11}$ L. Jankovič, D. Gournis, P. N. Trikalitis, I. Arfaoui, T. Cren, P. Rudolf, M. H. Sage, T. T. M. Palstra, B. Kooi, J. De Hosson, M. A. Karakassides, K. Dimos, A. Moukarika, and T. Bakas, Nano Lett. 6, 1131 (2006).

${ }^{12}$ N. Tombros, L. Buit, I. Arfaoui, T. Tsoufis, D. Gournis, P. N. Trikalitis, S. J. van der Molen, P. Rudolf, and B. J. van Wees, Nano Lett. 8, 3060 (2008).

${ }^{13}$ M. Savolainen, V. Touboltsev, P. Koppinen, K. P. Riikonen, and K. Arutyunov, Appl. Phys. A 79, 1769 (2004).

${ }^{14}$ M. Zgirski, K. P. Riikonen, V. Touboltsev, and K. Arutyunov, Nano Lett. 5, 1029 (2005).

${ }^{15}$ F. Altomare, A. M. Chang, M. R. Melloch, Y. Hong, and C. W. Tu, Phys. Rev. Lett. 97, 017001 (2006).

${ }^{16}$ K. Yu. Arutyunov, D. S. Golubev, and A. D. Zaikin, Phys. Rep. 464, 1 (2008).

${ }^{17}$ A. J. Leggett, Prog. Theor. Phys. 36, 901 (1966).

${ }^{18}$ Y. Tanaka, J. Phys. Soc. Jpn. 70, 2844 (2001).

${ }^{19}$ Y. Tanaka, Phys. Rev. Lett. 88, 017002 (2001).

${ }^{20}$ A. Gurevich and V. M. Vinokur, Phys. Rev. Lett. 90, 047004 (2003).

${ }^{21}$ E. Babaev, Phys. Rev. Lett. 89, 067001 (2002).

${ }^{22}$ E. Babaev, J. Jäykkä, and M. Speight, Phys. Rev. Lett. 103, 237002 (2009).

${ }^{23}$ J. M. Blatt and C. J. Thompson, Phys. Rev. Lett. 10, 332 (1963).

${ }^{24}$ A. A. Shanenko, M. D. Croitoru, M. Zgirski, F. M. Peeters, and K. Arutyunov, Phys. Rev. B 74, 052502 (2006).

${ }^{25}$ A. A. Shanenko, M. D. Croitoru, R. G. Mints, and F. M. Peeters, Phys. Rev. Lett. 99, 067007 (2007).
${ }^{26}$ A. A. Shanenko, M. D. Croitoru, and F. M. Peeters, Phys. Rev. B 78, 024505 (2008).

${ }^{27}$ J. E. Han and V. H. Crespi, Phys. Rev. B 69, 214526 (2004).

${ }^{28}$ K. Czajka, M. M. Maśka, M. Mierzejewski, and Z. Śledź, Phys. Rev. B 72, 035320 (2005).

${ }^{29}$ V. Vakaryuk, Phys. Rev. Lett. 101, 167002 (2008).

${ }^{30}$ F. Loder, A. P. Kampf, and T. Kopp, Phys. Rev. B 78, 174526 (2008).

${ }^{31}$ G. Schwiete and Y. Oreg, Phys. Rev. Lett. 103, 037001 (2009).

${ }^{32}$ P. G. de Gennes, Superconductivity of Metals and Alloys (Benjamin, New York, 1966).

${ }^{33}$ A. A. Shanenko and M. D. Croitoru, Phys. Rev. B 73, 012510 (2006).

${ }^{34}$ D. G. Naugle, J. W. Baker, and R. E. Allen, Phys. Rev. B 7, 3028 (1973).

${ }^{35}$ P. W. Anderson, J. Phys. Chem. Solids 11, 26 (1959).

${ }^{36}$ K. Tanaka and F. Marsiglio, Phys. Rev. B 62, 5345 (2000).

${ }^{37}$ A. A. Shanenko, M. D. Croitoru, and F. M. Peeters, Europhys. Lett. 76, 498 (2006); Phys. Rev. B 75, 014519 (2007).

${ }^{38}$ A. M. García-García, J. D. Urbina, E. A. Yuzbashyan, K. Richter, and B. L. Altshuler, Phys. Rev. Lett. 100, 187001 (2008).

${ }^{39}$ J. D. Shore, M. Huang, A. T. Dorsey, and J. P. Sethna, Phys. Rev. Lett. 62, 3089 (1989).

${ }^{40}$ T. Zhang, P. Cheng, W.-J. Li, Y.-J. Sun, G. Wang, X.-G. Zhu, K. He, L. Wang, X. Ma, X. Chen, Y. Wang, Y. Liu, H.-Q. Lin, J.-F. Jia, and Q.-K. Xue, Nat. Phys. 6, 104 (2010).

${ }^{41}$ Y. Chen, M. D. Croitoru, A. A. Shanenko, and F. M. Peeters, J. Phys.: Condens. Matter 21, 435701 (2009). 